\title{
Visualization of Neural DTI Vector Fields Using Line Integral Convolution
}

\author{
S.C.L. Deoni ${ }^{1}$, B.K. Rutt $^{1,2}$, and T.M. Peters ${ }^{1,2}$ \\ 1 Robarts Research Institute, University of Western Ontario, \\ 2 London Health Sciences Center, London, ON Canada \\ \{sdeoni, brutt, tpeters\}@imaging.robarts.ca
}

\begin{abstract}
Diffusion Tensor Imaging (DTI) provides voxel-wise information related to the local diffusion anisotropy. Recent research efforts have centered around the use of this information to infer the direction of local fiber bundles. Calculation of the diffusion tensor and corresponding principle diffusion direction voxel-wise throughout the imaged volume permits the use of tracking algorithms to reconstruct the fiber-bundle pathways. These algorithms are typically based on line propagation and provide results that visually resemble anatomical fiber dissections. Despite the success of these methods, they suffer several limitations, particularly within regions of decreased anisotropy, such as areas of grey matter and fiber crossing or branching. In this paper we present an alternative method of DTI data visualization, line integral convolution (LIC), which has several advantages over existing techniques, particularly, the ability to deal with noise and singularities within the vector field and areas of low anisotropy.
\end{abstract}

\section{Introduction}

The advent of diffusion-weighted imaging (DWI) and more recently, diffusion tensor imaging (DTI), of the brain has introduced the possibility of a noninvasive in-vivo means of visualizing fiber tracts and neural connectivity. DTI entails the collection of diffusion-weighted images, $S_{d}$, with diffusion encoding along at least six non-collinear directions, typically $(i, j, k)=(1,0,1),(-1,0,1)$, $(0,1,1),(0,1,-1),(1,1,0)$ and $(-1,1,0)$, along with a non diffusion-weighted image, $S_{o}$. The diffusion co-efficient in each of the six directions $\left(A D C_{d}\right)$ is calculated as:

$$
A D C_{d}=\frac{-\ln \left(\frac{S_{d}}{S_{o}}\right)}{b}
$$

where $b$ describes the amount of diffusion-weighting in the acquired images.

The diffusion co-efficients in the $\mathrm{X}, \mathrm{Y}, \mathrm{Z}, \mathrm{XY}, \mathrm{YZ}$ and $\mathrm{XZ}$ directions $\left(A D C_{x x}\right.$, $A D C_{y y}, A D C_{z z}, A D C_{x y}, A D C_{y z}$ and $A D C_{x z}$ ) describe the local diffusion tensor, and the principle direction of diffusion (PDD) is defined as the principle eigenvector of the $3 \times 3$ matrix. Determination of the PDD voxel-wise generates a vector field such as that illustrated in Fig. 1. In regions where diffusion along a 
single axis dominates, such as along a white matter tract, the associated fraction anisotropy $(F A)$, defined in Eq. 2, is high, while in regions where the diffusion is more isotropic, the $F A$ is lower.

$$
F A=\frac{3}{2} \times \frac{\sqrt{\left(\lambda_{1}-\lambda\right)^{2}+\left(\lambda_{2}-\lambda\right)^{2}+\left(\lambda_{3}-\lambda\right)^{2}}}{\sqrt{\lambda_{1}^{2}+\lambda_{2}^{2}+\lambda_{3}^{2}}}
$$

where $\lambda_{1}, \lambda_{2}$ and $\lambda_{3}$ are the principle, secondary and tertiary eigenvectors of the matrix and $\lambda$ is the average of the three.

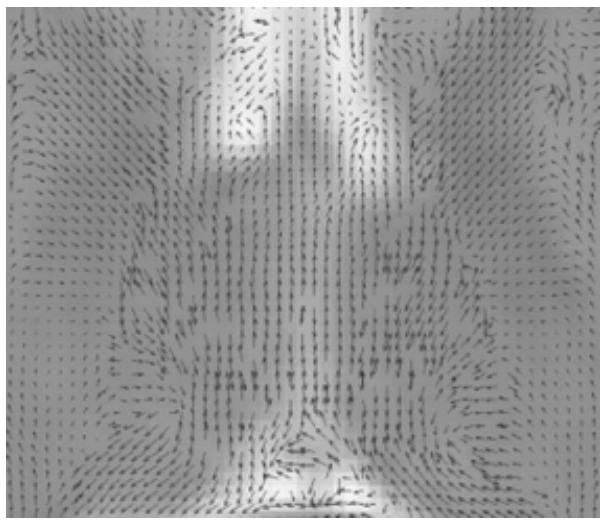

Fig. 1. Principle direction of diffusion within each voxel overlayed on the anatomical $T_{2}$-weighted image. Data corresponds to the deep brain.

Reconstruction of the fiber paths is a natural extension of DTI and Fig. 1 and provides insight into the three dimensional structure and connectivity of the data. Several methodologies of reconstruction, commonly referred to as tractography, have been developed [1-7] and can generally be classified as belonging to one of two groups: line propagation based methods and global statistical methods.

Line propagation (LP) is perhaps a more obvious and intuitive method of tractography in which a line or stream is extended from an initial seed point in the data along the local vector trajectories. The main difference between the various LP approaches [4-6], is the way in which the algorithm steps along the vector field from voxel to voxel. In general, the path is guided by the the assumption that the direction of the fiber path does not change abruptly (ie. it is smooth and continuous) [8]. A probability function is therefore used to determine the next step with straight paths preferred over large deviations. The primary disadvantage of this approach is that error is cumulative along the path.

Alternatively, tractography performed using global statistical methods such as simulated annealing [7], involve determination of the most-likely or leastcostly paths between two defined points (A and B) within the vector field. As 
such, all possible paths connecting point $\mathrm{A}$ with point $\mathrm{B}$ are calculated and assigned a probability or cost. The most-probable path from the calculated set is considered true. As with line propagation, these methods are guided by the smooth and continuous path assumption. The principle drawback of these methods is that a path will always be found connecting A and B, regardless of the anatomical validity of the path.

While tractography results from these methods are promising and visually resemble classic anatomical fiber dissections [9], they suffer from a number of limitations. In regions where the PDD is poorly defined or has a high associated uncertainty (low FA), such as in grey matter, along grey matter / white matter boundaries, or areas where fiber bundles cross or branch, these approaches can easily become side-tracked and wander off track. Additionally, when the propagating line encounters a branch, it is constrained to follow only one of the paths while ignoring the other.

Although not commonly considered a tractography method, line integral convolution (LIC) [10] is a widely-used approach for visualizing complex vector fields in two and three dimensions, such as those associated with turbulent flow. The LIC algorithm produces a texture representation of a vector field by locally blurring an input texture (usually white noise) with the vector field. An example of LIC is illustrated in Fig. 2 where we have applied the algorithm to a sinusoidal vector field.

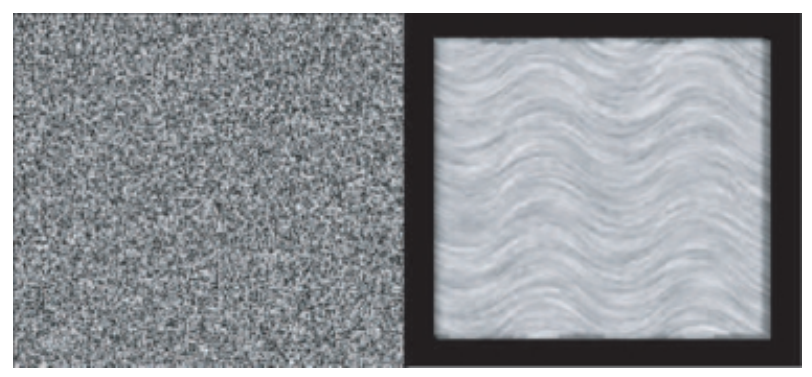

Fig. 2. The input white noise image on the left is blurred with the sinusoidal vector field using the LIC algorithm to produce the image on the right.

Areas of the vector field that are coherent and continuous are easily followed within the LIC result, via patterns of correlation in the output texture.

Despite its widespread use in computational fluid dynamics [11] LIC has not yet been adopted within the medical imaging community for visualization of DTI vector fields or reconstruction of neuronal fiber tracts. This is surprising since the LIC method offers several advantages over both line propagation and global statistical methods, particularly when considering its relative immunity to noise within the vector field. Since the LIC result is produced by locally blurring the noise image with the vector field at each point, small deviations in the vector field do not significantly influence the result. 
Areas of fiber branching and crossing are also handled well with LIC. While conventional line propagation methods limit the path to following only one branch or the other, LIC blurs, or smears, the texture along both paths. Additionally, since LIC takes advantage of the human visual system's ability to recognize and follow patterns [12], areas where fiber bundles cross are easily interpreted and the continuity of the paths inferred. This phenomenon is illustrated in Fig. 3. In a traditional tractography result, streamtubes propagated from the left or right would tend to change directions and follow the vertical path in the center.

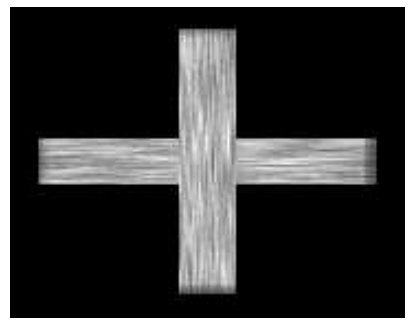

Fig. 3. LIC result of a vector field in which two paths cross. The human visual system is able to recognize and follow both paths.

In this paper we present a simplified version of the LIC algorithm for use with DTI data. We show that the LIC approach is a quick, intuitive and effective method of visualizing DTI data and produces qualitative results similar to conventional tractography methods.

\section{Methods}

\subsection{Simplified LIC Algorithm}

The LIC algorithm we present is a simplification of that originally presented by Cabral and Leedom in [10]. In the complete implementation, output pixel intensities are generated by a one-dimensional convolution of a filter kernel and the noise input texture along the local streamline through each pixel in the vector field. Mathematically, this is given by

$$
S I_{\text {out }}(i, j)=\sum_{p \subset \tau} S I_{\text {in }}(p) \cdot h(p)
$$

where

$\tau=$ the set of cells along the streamline within a set distance from point $(\mathrm{i}, \mathrm{j})$, shown in Fig. 4

$S I_{\text {in }}(p)=$ input noise texture at cell $p$ 
$h(p)=\int_{\alpha}^{\beta} k(w) d w$

$\alpha=$ the length of the streamline from $(\mathrm{i}, \mathrm{j})$ to the entrance of cell $p$

$\beta=$ the length of the streamline from $(\mathrm{i}, \mathrm{j})$ to the exit of cell $p$

$\mathrm{k}(\mathrm{w})$ is the convolution filter function.

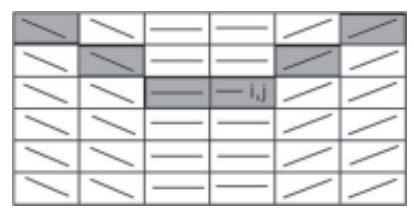

Fig. 4. Example Vector field showing the streamline through point $(\mathrm{i}, \mathrm{j})$.

In our adaptation of the algorithm, we assume the fiber bundles follow a smooth and continuous path. Therefore, the streamline through each point $(\mathrm{i}, \mathrm{j})$ in the vector field can be approximated as the tangent to the vector in $(i, j)$, Fig. 5. This simplification is introduced to help avoid singularities within the DTI data and to smooth out areas of noise. The vector field derived from DTI data is not a tradition vector field in that the absolute direction of the eigenvalues is not defined. Following the tangent of each vector reduces the difficulity associated with dealing with this property. Comparison of our implementation with the complete LIC algorithm (Fig. 6) shows this simplification introduces no visual difference between the results.

Additionally, we modify the input noise texture by first multiplying it with the normalized fractional anisotropy (FA) map. This has the effect of enhancing regions of high anisotropy, such as the major white matter tracts of the corpus callosum, in the resulting output texture and decreasing regions of low anisotropy, such as grey matter regions within the cortex.

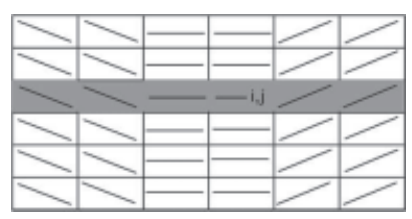

Fig. 5. Simplification of the LIC algorithm. The convolution is calculated along the tangent to $(\mathrm{i}, \mathrm{j})$ rather than along the streamline through $(\mathrm{i}, \mathrm{j})$

\subsection{In vivo Imaging}

Axially oriented images were acquired of a human volunteer using a diffusionweighted echo planar imaging (dwEPI) sequence with diffusion encoding along 


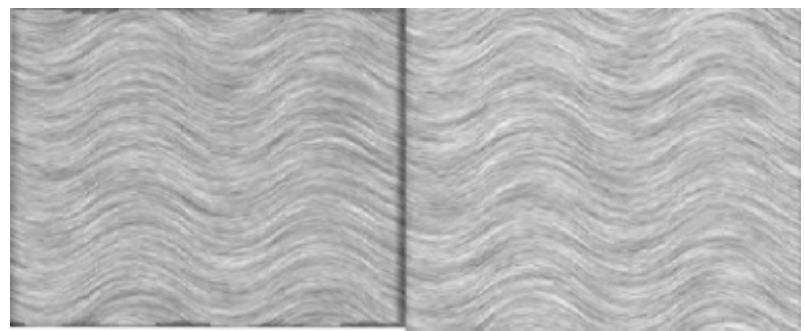

Fig. 6. Comparison of our simplified LIC approach (left) with the full algorithm (right). No visual difference can be seen between the two results.

six directions. Specific imaging parameters were as follows: $25 \mathrm{~cm}^{2} \times 5 \mathrm{~cm}$ field of view, $256 \times 128 \times 25$ matrix, $T E=67 \mathrm{~ms}, T R=10,000 \mathrm{~ms}$ and $b=600 \mathrm{~s} / \mathrm{mm}^{2}$.

The PDD and FA were calculated voxel-wise and the $3 \mathrm{D}$ vector data projected onto the X-Y plane. The vector data were limited to two dimensions for simplicity and ease of presentation.

\subsection{Tractography}

LIC and probability-based LP tractography [4] were performed on the data and visually compared. Seed points for the LP method were placed in every second pixel in both X and Y directions. Propagation of the streamline was terminated when the path entered a cell with a fractional anisotropy lower than a predetermined threshold of 0.2 .

\section{Results}

Results of the LIC and LP streamline algorithms are shown in Fig. 7. Paths corresponding to the major white matter tracts of the corpus callosum and splenium can be seen in both images as well as smaller projections within the cortex. Total processing time for the LIC result was less than 10 seconds, while the streamline analysis required 5 minutes on a Macintosh $1 \mathrm{GHz}$ G4.

An enlargement of the deep brain portion of the LIC result is shown in Fig. 8 and demonstrates distinguishable paths within the thalamus. Fiber tracking within gray matter structures has traditionally been avoided due to the low $F A$ of the regions, and the poor results obtained with LP streamline methods. The LIC result demonstrates fiber structure within the thalamus and the algorithm's ability to deal with the vector field noise.

\section{Conclusions}

Visualization of two and three dimensional vector fields is a challenging problem. Traditional methods of visualization, such as drawing arrows within each 

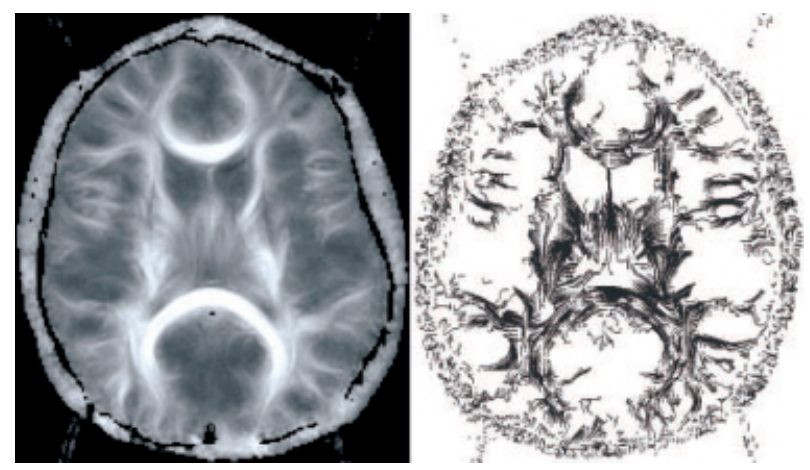

Fig. 7. Results of the LIC (left) and probability-based tractography (right) algorithms.

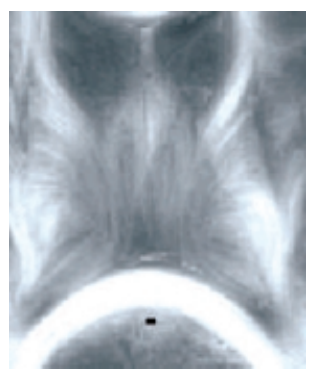

Fig. 8. Enlargement of the deep brain region in the LIC result showing distinguishable paths within the thalamus.

voxel or generating streamlines throughout the vector field, can produce confusing images, especially in areas where the PDD changes quickly. Additionally, streamline based methods are intrinsically sensitive to noise within the vector field.

In this paper we have evaluated the use of LIC for visualizing human brain DTI data and have compared the results with those of a conventional line propagation tractography method. We have found that LIC is a powerful technique for visualizing the complex vector fields associated with DTI and offers several advantages over the more conventional tractography. Among these advantages are the computational speed of the algorithm. The texture can be computed either slice-by-slice or from the full 3D data allowing easy visualization from any orientation. 3D results can be displayed using three orthogonal planes, however, this hinders the ability to view tracts running in non-orthogonal directions. Volume segmentation and colour augmentation offer a more intuitive and appealing method of display. Animation may also be used to visualize the $3 \mathrm{D}$ data set, by serially looping multiple LIC results calculated with the same noise input function but shifted in phase, the perception of flow is seen in the 3D data. An additional advatange is the algorithms robustness to noise within the vector 
field. This robustness negates the need for complex data smoothing approaches. The algorithm performs well even in areas of low anisotropy, and reveals grey matter structure not seen with conventional tractography approaches.

\section{Acknowledgments.}

The authors wish to thank Drs. Derek Jones and Yi-Fen Yen for their helpful discussions and insight. Funding for this work was provided by the Canadian Institutes for Health Research (grants MT-11540 and GR-14973), the Canadian Foundation for Innovation, the University of Western Ontario and General Electric Medical Systems.

\section{References}

1. S. Pajevic, A. Aldroubi, J. Duda, and P.J. Basser. A continuous tensor field approximation of discrete dt-mri data for extracting microstructural and architectural features of tissues. Journal of Magnetic Resonance, 154:85-100, 2002.

2. P.J. Basser, S. Pajevic, C. Peropaoli, J. Duda, and A. Aldroubi. In vivo fiber tractography using dt-mri data. Magnetic Resonance in Medicine, 44:625-632, 2000 .

3. C. Gossl, L. Fahrmeir, B. Putz, L.M. Auer, and D.P. Auer. Fiber tracking from dti using linear state space models: Detectability of the pyramidal tract. NeuroImage, 16:378-388, 2002.

4. JD Tournier, F Calamant, DG Gadian, and A Connelly. A novel fiber-tracking technique: front evolution using a fiber orientation probability density function. In Proc. of the International Society of Magnetic Resonance in Medicine, 2002.

5. M Lazar and AI Alexander. White matter tractography using random vector (wave) perturbation. In Proc. of the International Society of Magnetic Resonance in Medicine, 2002.

6. M Bjornemo, A Brun, R Kikinis, and CF Westin. Regularized stochastic white matter tractography using diffusion tensor mri. In Proc. Medical Image Computing and Computer Assisted Intervention, 2002.

7. DS Tuch, MR Wiegell, TG Reese, JW Belliveau, and V Weeden. Measuring corticocortical connectivity matricies with diffusion spectrum imaging. In Proc. of the International Society of Magnetic Resonance in Medicine, 2001.

8. S Mori, WK Kaufmann, GD Pearlson, BJ Crain, B Stieltjes, M Solaiyappan, and PCM van Zijl. In vivo visualization of human neural pathways by mri. Annals of Neurology, 47:412-414, 2000.

9. S Mori, WK Kaufmann, C Stieljes, L Amodei, K Fredrickson, GD Pearlson, ER Melhem, M Solaiyappan, GV Raymond, HW Moser, and PCM van Zijl. Imaging cortical association tracts in the human brain using diffusion-tensor based axonal tracking. Magnetic Resonance in Medicine, 47:215-223, 2002.

10. B Cabral and C Leedom. Imaging vector fields using line integral convolution. In Computer Graphics Proceedings '93, ACM SIGGRAPH, 1993.

11. HW Shen and DL Kao. A new line integral convolution algorithm for visualizing time-varying flow fields. IEEE Transactions on Visualization and Computer Graphics, pages 98-108, 1998.

12. WD Ellis. A source book of Gestalt psychology. Harcourt, Brace and Co, 1939. 